\title{
MHD convection flow in a constricted channel
}

\author{
M. Tezer-Sezgin and Merve Gürbüz
}

\begin{abstract}
We consider the steady, laminar, convection flow in a long channel of $2 D$ rectangular constricted cross-section under the influence of an applied magnetic field. The Navier-Stokes equations including Lorentz and buoyancy forces are coupled with the temperature equation and are solved by using linear radial basis function (RBF) approximations in terms of the velocity, pressure and the temperature of the fluid. RBFs are used in the approximation of the particular solution which becomes also the approximate solution of the problem. Results are obtained for several values of Grashof number $(G r)$, Hartmann number $(M)$ and the constriction ratios $(C R)$ to see the effects on the flow and isotherms for fixed values of Reynolds number and Prandtl number. As $M$ increases, the flow is flattened. An increase in $G r$ increases the magnitude of the flow in the channel. Isolines undergo an inversion at the center of the channel indicating convection dominance due to the strong buoyancy force, but this inversion is retarded with the increase in the strength of the applied magnetic field. When both Hartmann number and constriction ratio are increased, flow is divided into more loops symmetrically with respect to the axes.
\end{abstract}

\section{Introduction}

Flow and heat transfer from irregular surfaces have attracted the considerable interest of many researchers due to the wide range of engineering applications

Key Words: MHD convection flow, RBF, constricted channel.

2010 Mathematics Subject Classification: Primary 76W05, 76R99; Secondary 65M70.

Received: November, 2016.

Revised: March, 2017.

Accepted: June, 2017. 
such as micro-electronic devices, flat-plate solar collectors, geophysical applications, cooling system etc. Kolodziej et al. [1] have solved laminar convection flow in a wavy channel using the method of fundamental solution and RBFs. In [2], the mixed convection heat transfer in a lid-driven cavity with a sinusoidal wavy bottom surface is analyzed by using finite element formulation based on the Galerkin method. They showed that average Nusselt number increases as the amplitude of the wavy surface and Reynolds number increase. Transient convective heat transfer for Rayleigh Benard convection flow is studied by Cetindag et al. [3] in an air-filled shallow enclosure. The magnetic effect on mixed convection flow in a lid-driven cavity has been added by Nasrin et al. [4]. MHD mixed convection boundary layer flow on inclined wavy plate has been analyzed by Wang et al. [5]. They indicated that the heat transfer rate and the skin-friction coefficient increase with an inclined magnetic field. Colaço et al. [6] have applied multiquadratic RBF approximation to convection flow in a square cavity under the influence of horizontal magnetic field. Computations are carried out for several Hartmann number and Grashof number values at fixed $\operatorname{Pr}=0.71$. The effects of magnetic field on free convection flow is also analyzed by Lo [7]. The numerical results are obtained for different values of Hartmann number by taking $P r=0.71$. Mößner et al. [8] have studied the effects of stationary magnetic fields on $3 \mathrm{D}$ natural convection in liquid metals. They showed that the number of convection rolls in the cavity increases as Hartmann number increases or Rayleigh number decreases.

In this study, we apply the linear polynomial RBF approximation to MHD convection flow in a constricted rectangular enclosure. A uniform magnetic field is applied $x$ - or $y$-direction. In [9], simulation Stokes flow $(\operatorname{Re}<<1)$ in a constricted channel with a moving left wall in the presence of vertically applied magnetic field is presented. The aim of this study is to investigate the effect of magnetic field, buoyancy force, constriction and the length of the channel on the flow and the temperature of the fluid. The results are depicted in terms of stream function, vorticity, temperature, and the pressure of the fluid.

\section{Mathematical formulation}

The steady convection flow of an electrically conducting fluid is considered in a constricted channel. Flow is subjected to a uniform magnetic field. The physical properties of the fluid are assumed to be constant except for the buoyancy term in the momentum equations [10]. Induced magnetic field, joule heating, viscous dissipation and volumetric energy are neglected due to the small magnetic Reynolds number, small electrical conductivity, absence of internal energy and other sources of volumetric energy release, respectively. Thus, 
the continuity, momentum and energy equations are given in non-dimensional form as

$$
\begin{gathered}
\frac{\partial u}{\partial x}+\frac{\partial v}{\partial y}=0 \\
\operatorname{Re}\left(u \frac{\partial u}{\partial x}+v \frac{\partial u}{\partial y}\right)=-\frac{\partial p}{\partial x}+\nabla^{2} u+M^{2}\left(-u H_{y}{ }^{2}+v H_{x} H_{y}\right) \\
\operatorname{Re}\left(u \frac{\partial v}{\partial x}+v \frac{\partial v}{\partial y}\right)=-\frac{\partial p}{\partial y}+\nabla^{2} v+M^{2}\left(u H_{x} H_{y}-v H_{x}{ }^{2}\right)+\frac{G r}{R e} T \\
\frac{1}{P r} \nabla^{2} T=\operatorname{Re}\left(u \frac{\partial T}{\partial x}+v \frac{\partial T}{\partial y}\right)
\end{gathered}
$$

by using the dimensionless variables defined as

$$
\begin{aligned}
& \mathbf{x} \rightarrow \mathbf{x} L, \quad \mathbf{u} \rightarrow \mathbf{u} U_{0}, \quad \mathbf{H} \rightarrow \mathbf{H} H_{0}, \\
& p \rightarrow p \rho \nu U_{0} / L, \quad T-T_{\text {cold }} \rightarrow T\left(T_{\text {hot }}-T_{\text {cold }}\right)
\end{aligned}
$$

where $\mathbf{u}=(u, v), p, \mathbf{H}=\left(H_{x}, H_{y}\right)$ and $T$ are the velocity, pressure, the magnetic field and the temperature of the fluid, respectively.

The non-dimensional parameters are the Reynolds number $R e=L U_{0} / \nu$, the Hartmann number $M=L \mu H_{0} \sqrt{\sigma / \rho \nu}$, the Prandtl number $\operatorname{Pr}=\rho c_{p} \nu / \lambda$ and the Grashof number $G r=g \beta\left(T_{h o t}-T_{\text {cold }}\right) L^{3} / \nu^{2}$. Here, $H_{0}=\left(H_{x}{ }^{2}+\right.$ $\left.H_{y}{ }^{2}\right)^{1 / 2}, U_{0}=\sqrt{g \beta L\left(T_{\text {hot }}-T_{\text {cold }}\right)}, L, \nu, \sigma, \mu, \rho, c_{p}, \beta$ and $\lambda$ are the externally applied magnetic field intensity, the characteristic velocity, the characteristic length, kinematic viscosity, electrical conductivity, magnetic permeability, the density, specific heat, thermal expansion coefficient and the thermal conductivity of the fluid, respectively.

\subsection{Horizontal or vertical external magnetic field}

When the magnetic field is applied vertically, $\mathbf{H}_{\mathbf{0}}=\frac{\mathbf{H}}{H_{0}}=(0,1)$, the equations (1)-(4) can be written as

$$
\begin{gathered}
\frac{\partial u}{\partial x}+\frac{\partial v}{\partial y}=0 \\
\operatorname{Re}\left(u \frac{\partial u}{\partial x}+v \frac{\partial u}{\partial y}\right)=-\frac{\partial p}{\partial x}+\nabla^{2} u-M^{2} u \\
\operatorname{Re}\left(u \frac{\partial v}{\partial x}+v \frac{\partial v}{\partial y}\right)=-\frac{\partial p}{\partial y}+\nabla^{2} v+\frac{G r}{R e} T \\
\frac{1}{P r} \nabla^{2} T=\operatorname{Re}\left(u \frac{\partial T}{\partial x}+v \frac{\partial T}{\partial y}\right)
\end{gathered}
$$


In the cross-section of the channel the flow is regarded as two-dimensional so that the stream function $\psi, u=\frac{\partial \psi}{\partial y}, v=-\frac{\partial \psi}{\partial x}$, and the vorticity, $\omega=$ $\frac{\partial v}{\partial x}-\frac{\partial u}{\partial y}$ can be defined.

Then, the two-dimensional MHD convection flow is represented with Poisson's type equations in terms of velocity components, stream function, temperature, vorticity and pressure

$$
\begin{gathered}
\nabla^{2} u=-\frac{\partial \omega}{\partial y}, \quad \nabla^{2} v=\frac{\partial \omega}{\partial x} \\
\nabla^{2} \psi=-\omega \\
\nabla^{2} T=\operatorname{Pr} \operatorname{Re}\left(u \frac{\partial T}{\partial x}+v \frac{\partial T}{\partial y}\right) \\
\nabla^{2} \omega=\operatorname{Re}\left(u \frac{\partial \omega}{\partial x}+v \frac{\partial \omega}{\partial y}\right)-M^{2} \frac{\partial u}{\partial y}-\frac{G r}{\operatorname{Re}} \frac{\partial T}{\partial x} \\
\nabla^{2} p=-2 \operatorname{Re}\left(\frac{\partial v}{\partial x} \frac{\partial u}{\partial y}-\frac{\partial u}{\partial x} \frac{\partial v}{\partial y}\right)-M^{2} \frac{\partial u}{\partial x}+\frac{G r}{\operatorname{Re}} \frac{\partial T}{\partial y} .
\end{gathered}
$$

When the magnetic field is in the $x$-direction, $\mathbf{H}_{\mathbf{0}}=(1,0)$, the vorticity and the pressure equations are altered only by changing the terms $-M^{2} \frac{\partial u}{\partial y}$, $-M^{2} \frac{\partial u}{\partial x}$ with $M^{2} \frac{\partial v}{\partial x},-M^{2} \frac{\partial v}{\partial y}$.

The problem geometry and the boundary conditions are shown in the Figure 1 . The vorticity boundary values are obtained from the stream function equation by using the finite difference method including interior values of stream function. The middle section of the enclosure is symmetrically constricted using functions $f_{b}$ and $f_{t}$, which are the vertical coordinates of the bottom and top walls, respectively. These functions are given as,

$$
f_{b}=\frac{1}{2} h(1+\cos (2 \pi(x-A / 2) / A)), \quad f_{t}=1-f_{b}
$$

where $A$ is the length of the channel. The constriction ratio $(C R)$ of the enclosure is defined as $C R=2 h \times 100$. 


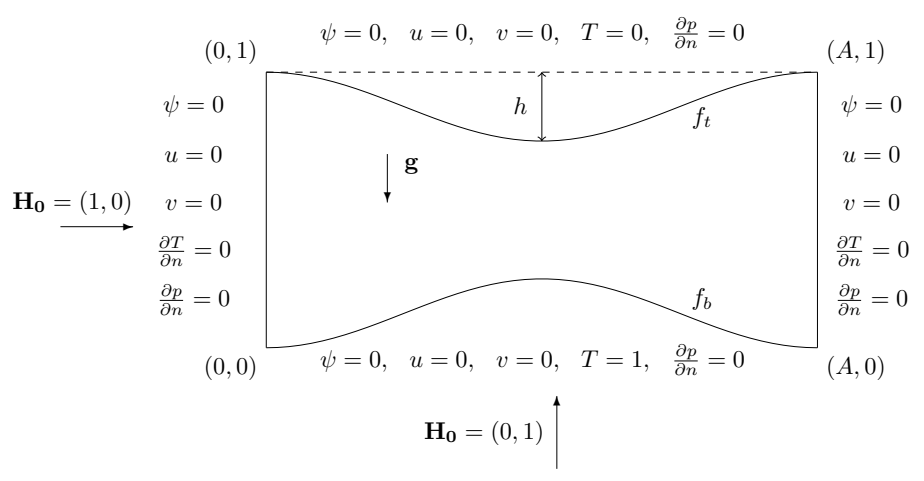

Figure 1: Schematic of the rectangular enclosure with constriction and the boundary conditions

\section{RBF approximation}

In the radial basis function approximation [11], the right hand side of the partial differential equation $L u(x, y)=f(x, y)$ and the particular solution $\hat{u}$ can be written in a finite series of $\operatorname{RBFs}\left\{\varphi_{j}\right\}$ and $\left\{\Psi_{j}\right\}$ as

$$
f(x, y)=\sum_{j=1}^{n} a_{j} \varphi_{j}(r), \quad \hat{u}(x, y)=\sum_{j=1}^{n} a_{j} \Psi_{j}(r) \quad(x, y) \in \Omega
$$

where $r=\sqrt{\left(x-x_{j}\right)^{2}+\left(y-y_{j}\right)^{2}}$ is the Euclidean distance. $\Psi_{j}$ 's are linked to $\varphi_{j}$ 's through $L \Psi_{j}(r)=\varphi_{j}(r)$ and $n$ is the number of unknown coefficients. $\hat{u}$ is forced to satisfy the boundary condition $B u=g$ as

$$
\sum_{j=1}^{n} a_{j} B \Psi_{j}(r)=g(x, y), \quad(x, y) \in \partial \Omega .
$$

The coefficients $a_{j}$ in the approximation (14) are determined by taking $N$ collocation points $\left(x_{i}, y_{i}\right)$ on the boundary and $K$ points in the interior of the domain as

$$
\begin{aligned}
& \sum_{j=1}^{n} a_{j} B \Psi_{j}\left(r_{k}\right)=g\left(x_{k}, y_{k}\right), \quad 1 \leq k \leq N \quad \text { and } \\
& \sum_{j=1}^{n} a_{j} \varphi_{j}\left(r_{l}\right)=f\left(x_{l}, y_{l}\right), \quad 1+N \leq l \leq n \quad(n=N+K)
\end{aligned}
$$

which give one linear system $[A]\{a\}=\{b\}$ for the solution vector $\{a\}=\left[\begin{array}{lll}a_{1} & \cdots & a_{n}\end{array}\right]^{t}$. The coefficient matrix and the right hand side vector 
are given as

$$
[A]=\left[\begin{array}{cccc}
B \Psi_{1}\left(r_{1}\right) & B \Psi_{2}\left(r_{1}\right) & \cdots & B \Psi_{n}\left(r_{1}\right) \\
\vdots & \vdots & \ddots & \vdots \\
B \Psi_{1}\left(r_{N}\right) & B \Psi_{2}\left(r_{N}\right) & \cdots & B \Psi_{n}\left(r_{N}\right) \\
\varphi_{1}\left(r_{N+1}\right) & \varphi_{2}\left(r_{N+1}\right) & \cdots & \varphi_{n}\left(r_{N+1}\right) \\
\vdots & \vdots & \ddots & \vdots \\
\varphi_{1}\left(r_{n}\right) & \varphi_{2}\left(r_{n}\right) & \cdots & \varphi_{n}\left(r_{n}\right)
\end{array}\right]_{n \times n},\{b\}=\left[\begin{array}{c}
g\left(x_{1}, y_{1}\right) \\
\vdots \\
g\left(x_{N}, y_{N}\right) \\
f\left(x_{N+1}, y_{N+1}\right) \\
\vdots \\
f\left(x_{n}, y_{n}\right)
\end{array}\right]_{n \times 1}
$$

Solution of this system gives the coefficients $a_{j}$ 's, $1 \leq j \leq n$, and $\hat{u}(x, y)=\sum_{j=1}^{n} a_{j} \Psi_{j}(r)$.

In this study, $L=\nabla^{2}$ is the Laplace operator and the unknown variables are $u, v, \psi, \omega, T$ and $p$. Since the Poisson's type equations (9)-(12) are coupled, they are solved iteratively by using polynomial $\operatorname{RBFs}(\varphi(r)=1+r)$ and $\Psi(r)=\frac{r^{2}}{4}+\frac{r^{3}}{9}$ from $\nabla^{2} \Psi=\varphi$. First, the velocity components and the stream function are obtained with an initial estimate of vorticity from the equations (9)-(10). Then, the temperature equation (11) is solved with the obtained values of velocity components and the initial estimate of the temperature. After the unknown vorticity boundary values are obtained from the stream function equation by using finite difference scheme including interior stream function values [12], the vorticity equation (12) is solved by using the new values of velocity components and the temperature. The iteration continues until a preassigned tolerance $(\epsilon)$ is reached between two successive iterations

$$
\left\|z^{m+1}-z^{m}\right\|_{\infty}=\max _{1 \leq i \leq n}\left|z_{i}^{m+1}-z_{i}^{m}\right|<\epsilon
$$

where $z_{i}$ denotes $u, v, \psi, \omega$ or $T$ at the collocation point $\left(x_{i}, y_{i}\right)$. Then, we solve pressure equation (13) by using converged values of $u, v$ and $T$. In each iteration, all the space derivatives of unknowns are approximated by coordinate matrix $\varphi$ as

$$
\frac{\partial D}{\partial x}=\frac{\partial \varphi}{\partial x} \varphi^{-1} D, \quad \frac{\partial D}{\partial y}=\frac{\partial \varphi}{\partial y} \varphi^{-1} D
$$

where $D$ denotes $u, v, \omega$ and $T$. 


\section{Numerical results}

The results are simulated in terms of stream function, vorticity, temperature and pressure of the fluid for several values of the Hartmann number, Grashof number and the constriction ratio of the channel for fixed $R e=100$ and $\operatorname{Pr}=0.71$. The convergence tolerance is generally $\epsilon=10^{-7}$ and $\epsilon=10^{-11}$ for larger value of parameter $G r$ and $M$. We take $N=80,72$ and 70 uniformly distributed boundary points with sufficient number of interior points for the length of channel $A=1,2$ and 4 , respectively. The aim of the study is to analyze the impacts of the Hartmann number, Grashof number on the MHD convection flow in the constricted channel.

\subsection{Magnetic field in the $y$-direction}

MHD convection flow equations (9)-(13) are solved iteratively when the magnetic field is applied vertically. We take Grashof number $5 \times 10^{3} \leq G r \leq$ $5 \times 10^{4}$, Hartmann number $0 \leq M \leq 25$ and the constriction ratio $0 \% \leq$ $C R \leq 40 \%$. The influence of the horizontal length of the channel is also analyzed by taking $A=1,2,4$.

The proposed numerical procedure is validated first for the natural convection flow in non-constricted rectangular enclosure by taking $A=4, G r=$ $25 \times 10^{4}, M=0$ and $\operatorname{Pr}=0.01$ which corresponds to $R a=2500$. The flow and temperature behaviors are in well agreement with the ones given in Figures 5-6 in [13] which are obtained by using finite volume method.

Figure 2 shows the effect of Grashof number increase in the non-constricted square channel $(C R=0 \%)$ neglecting magnetic field $(M=0)$. As $G r$ increases, magnitude of the flow and pressure increases. When $G r>10^{4}$, the flow $(\psi, \omega)$ is separated into two loops symmetrically with respect to $x=0.5$. Convection dominance is observed but isotherms show symmetry with respect to $x=0.5$ as the flow bending at the center of the cavity for $G r>10^{4}$.

We analyze the influence of magnetic field on the flow, temperature and pressure behaviors for fixed $G r=10^{4}$ in Figure 3. As the intensity of the magnetic field increases, flow is flattened which is an expected behavior for MHD flow. Further increase in $M$ results in the retardation of the convection dominance which can be observed in isotherms as becoming straight lines parallel to the hot and cold walls. Similarly, pressure behavior is uniformly distributed in the channel. 


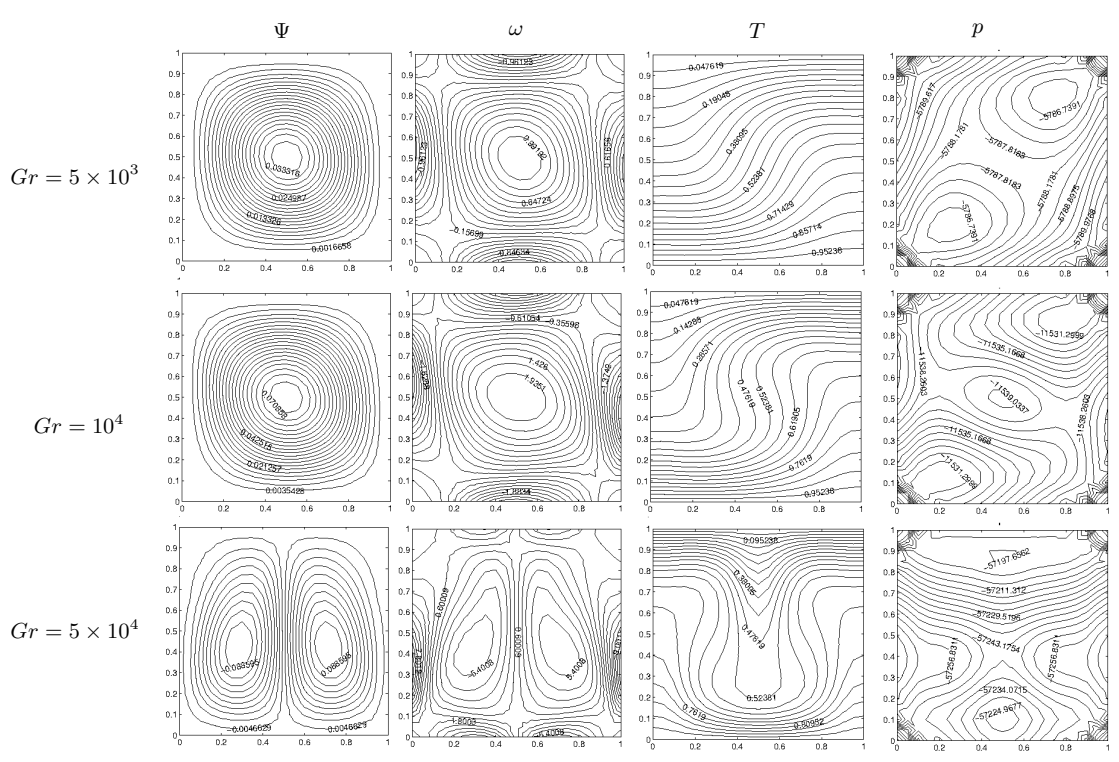

Figure 2: The effect of $G r$ on the flow, temperature and pressure for $M=0$ and $A=1$.

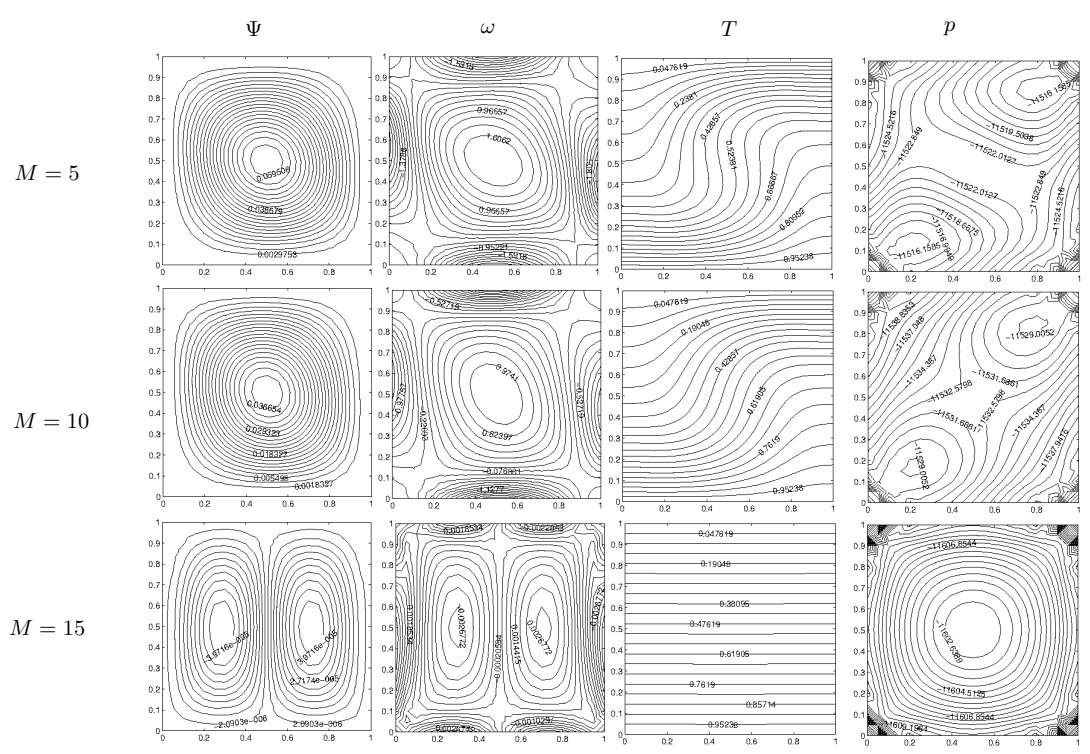

Figure 3: The effect of $M$ on the flow, temperature and pressure for $G r=10^{4}$ and $A=1$. 
Figures 4-5 depict the effect of magnetic field for conducting fluid in a non-constricted rectangular channel for increasing horizontal length $A=2,4$. When the length of the channel increases, the number of convection rolls (streamlines and vorticity loops) increases $A$ times and isotherms repeat its behavior symmetrically with respect to $A / 2$ line which can be seen from the first row of Figure 4 and Figure 5. Meantime boundary layer formation takes place in the flow on the walls parallel and perpendicular to applied magnetic field as $M$ increases. The same effect of increasing $M$ on the flow and temperature is observed as in the square channel (Figure 3).

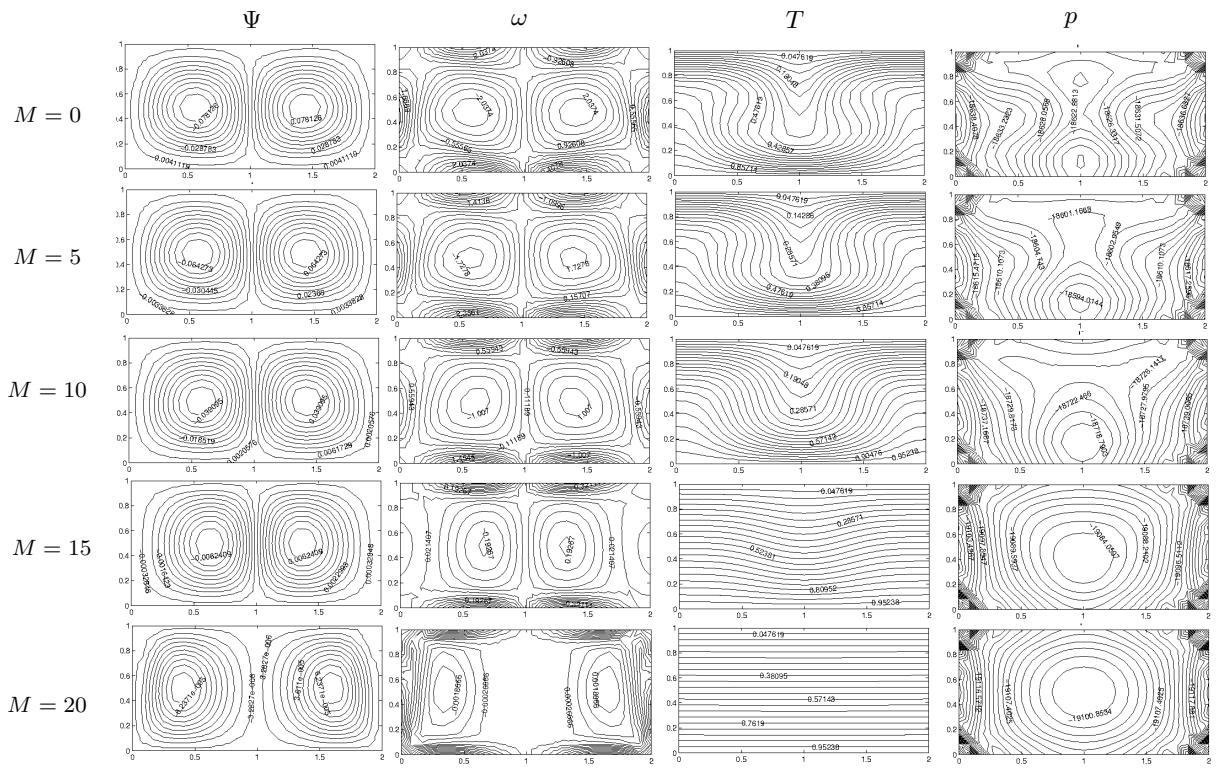

Figure 4: The effect of $M$ on the flow, temperature and pressure for $G r=10^{4}$ and $A=2$.

An increase in $M$ causes Hartmann layers near to the top and bottom walls and side layers parallel to applied magnetic field leaving the central part stagnant. When the Hartmann number value is reached to 15 , the number of rolls in stream function and vorticity also increases for $A=4$ as can be seen in Figure 5. 


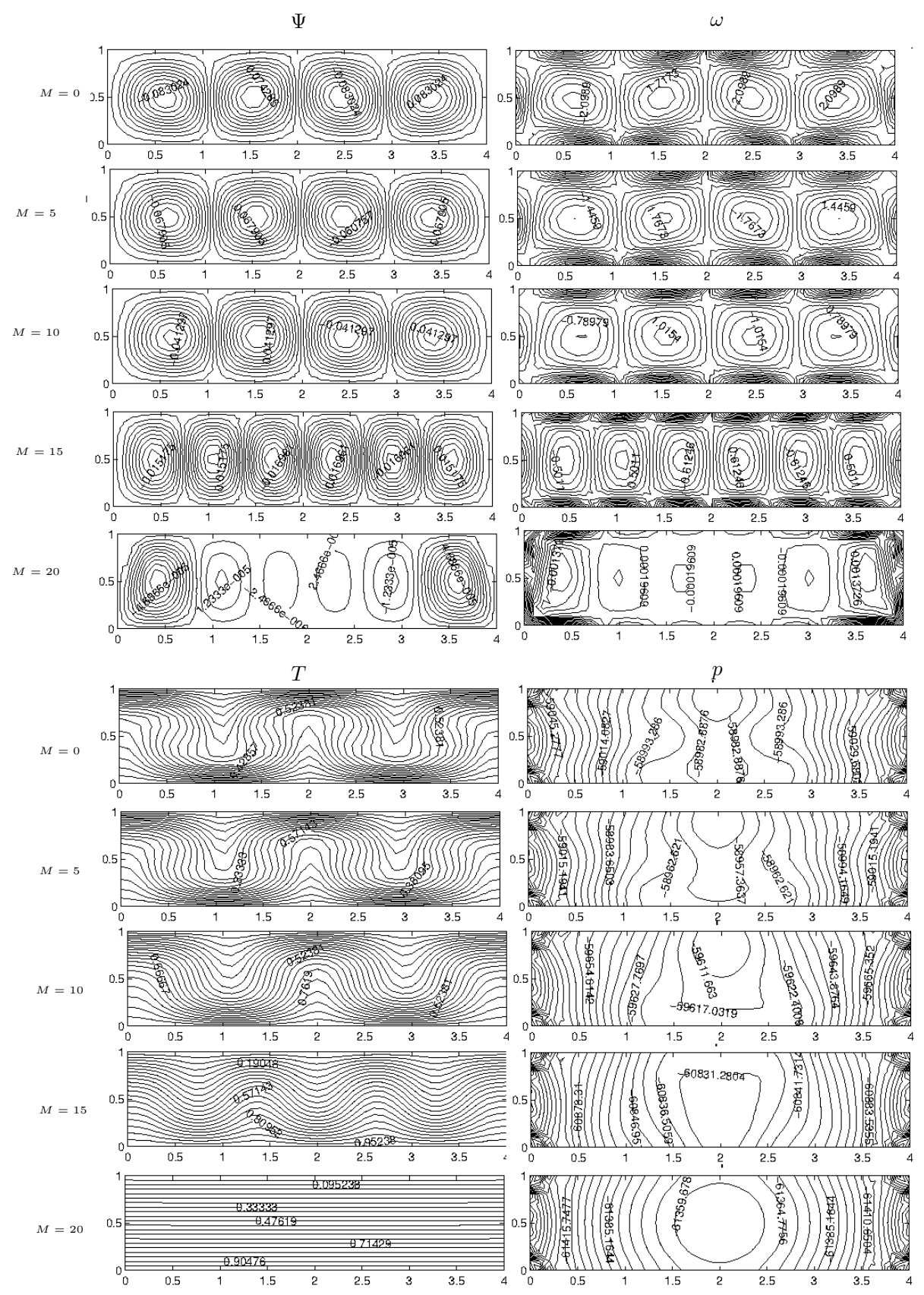

Figure 5: The effect of $M$ on the flow, temperature and pressure for $G r=10^{4}$ and $A=4$. 
The effect of constriction ratio on the convection flow under the effect of vertical magnetic field is shown in Figure 6 . There is a symmetry in the flow, temperature and pressure with respect to vertical centerline. As the constriction ratio increases, flow vortices at the constriction are started to be weakened and the fluid becomes almost stagnant at the middle of the channel. Fluid concentrates through the adiabatic walls due to the constriction. This causes the retardation of the convection in the constriction area. Similarly, the magnitude of the pressure is increased through the side walls.

For fixed $C R=40 \%$ we increase the Hartmann number to see the impact of magnetic field on MHD convection flow in a constricted channel with the length $A=4$. In Figure 7 , as $M$ increases magnitude of the flow decreases but the pressure increases as in the non-constricted channel in Figure 5. The increase in the magnitude of applied magnetic field first weakens and then increases the number of the center vortices in the flow $(M \leq 10)$. In constricted channel the division of the flow into symmetric vortices starts for a smaller value of $M \geq 10$ when we compare with the rectangular case and Hartmann layer formation starts. For $M \geq 15$ the flow vortices are symmetrically located with respect to vertical and horizontal centerlines. Isolines become completely diffusion dominated and distributed uniformly between the horizontal walls.

In Figure 8, we fix $C R=40 \%$ and increase $G r$ for $M=10$ and $M=20$ to analyze the effects of buoyancy force and magnetic force on the convection flow in a constricted channel. For a small effect of magnetic field, the increase in Gr destroys the symmetrically located four loops in the flow into two loops symmetric with respect to $x=1$. As $M$ increases, the symmetric behavior of the convection flow remains the same even the Gr increases up to $10^{4}$. Due to the increase in both constriction and Gr number the new vortices appear symmetrically at the center (constriction area) of the cavity. $\mathrm{Gr}$ increase results in bending in isotherms. 

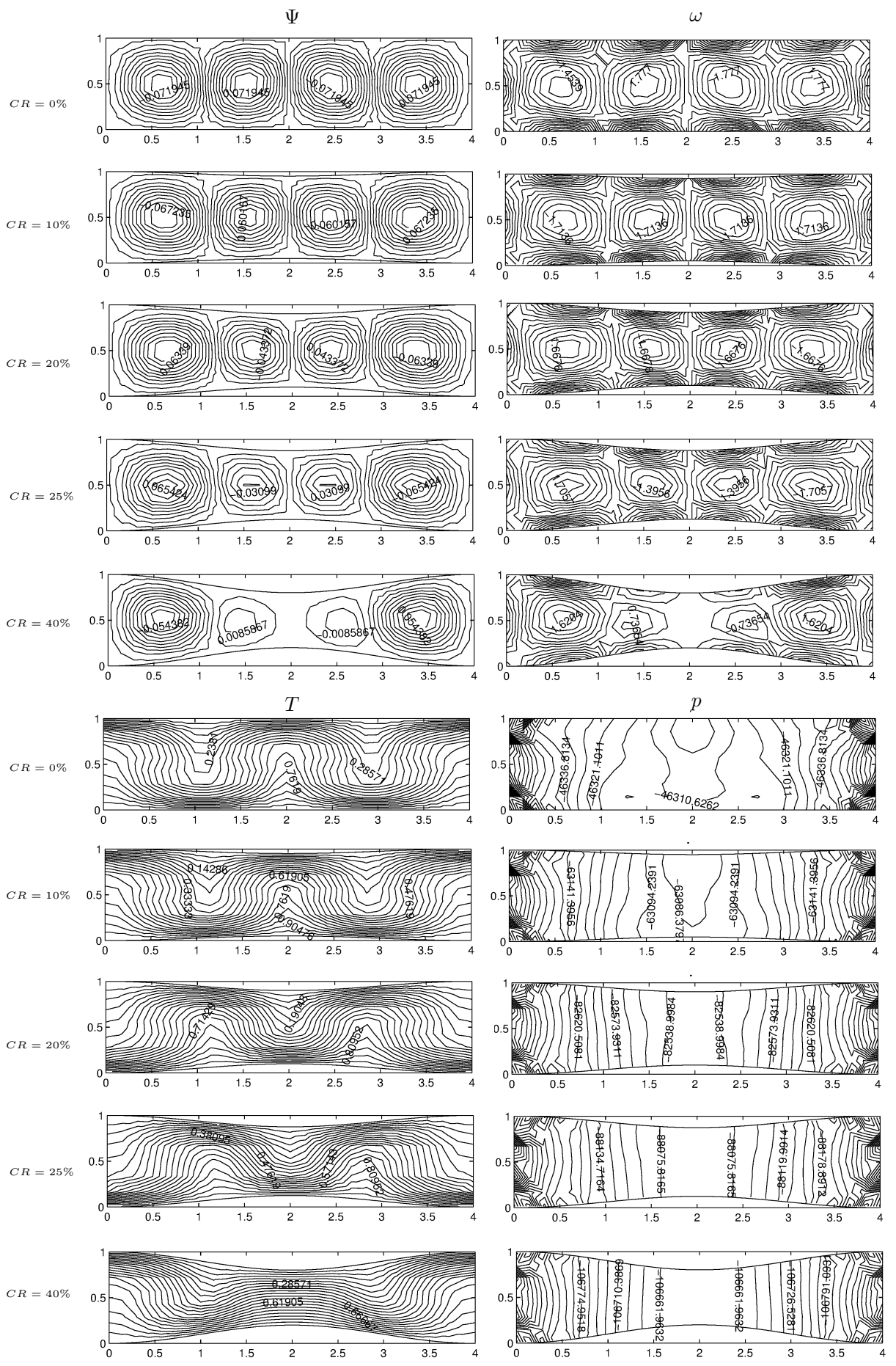

Figure 6: The effect of $C R$ on the flow, temperature and pressure for $G r=10^{4}$, $M=4$ and $A=4$. 

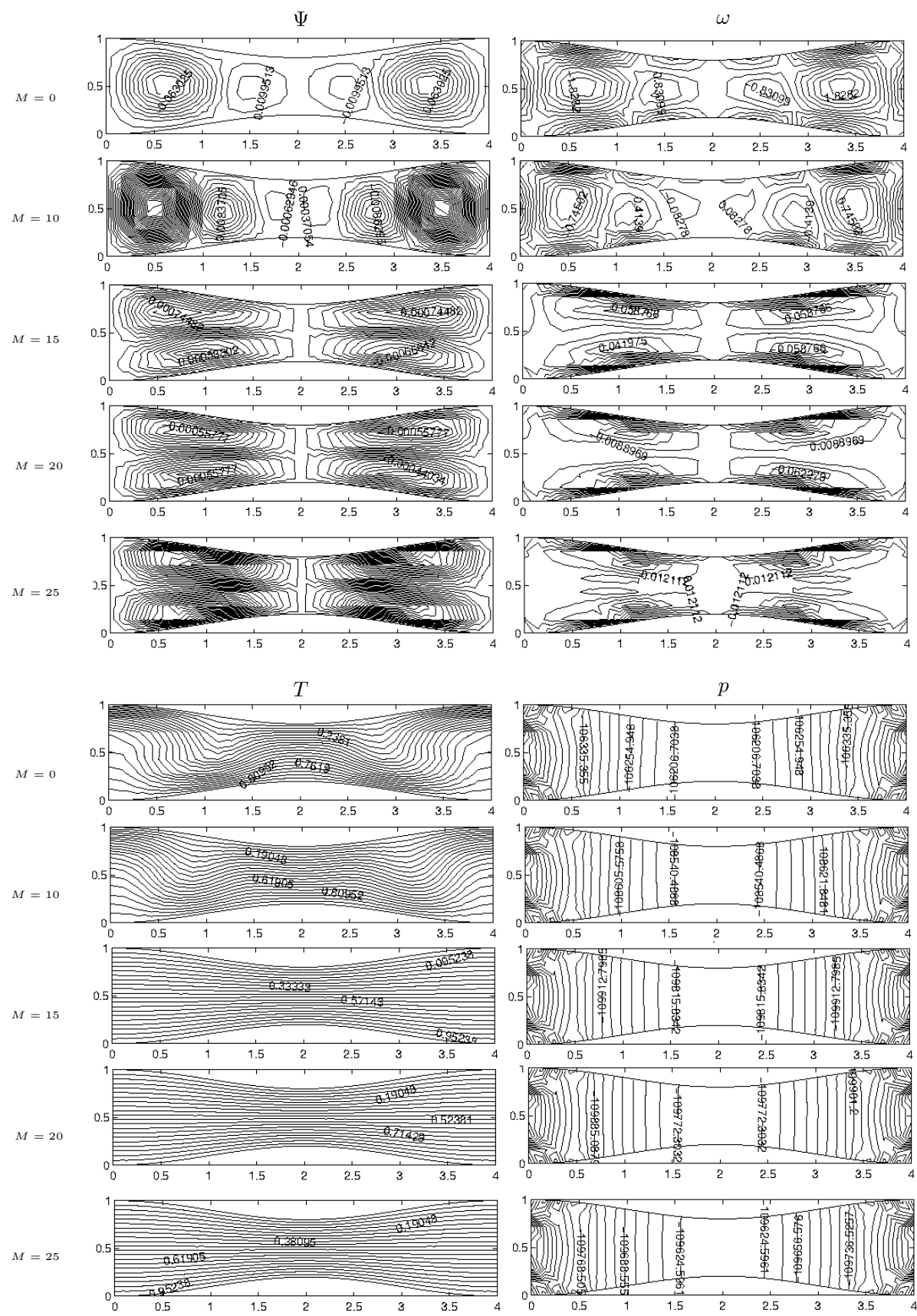

Figure 7: The effect of $M$ on the flow, temperature and pressure for $G r=10^{4}$, $C R=40 \%$ and $A=4$. 

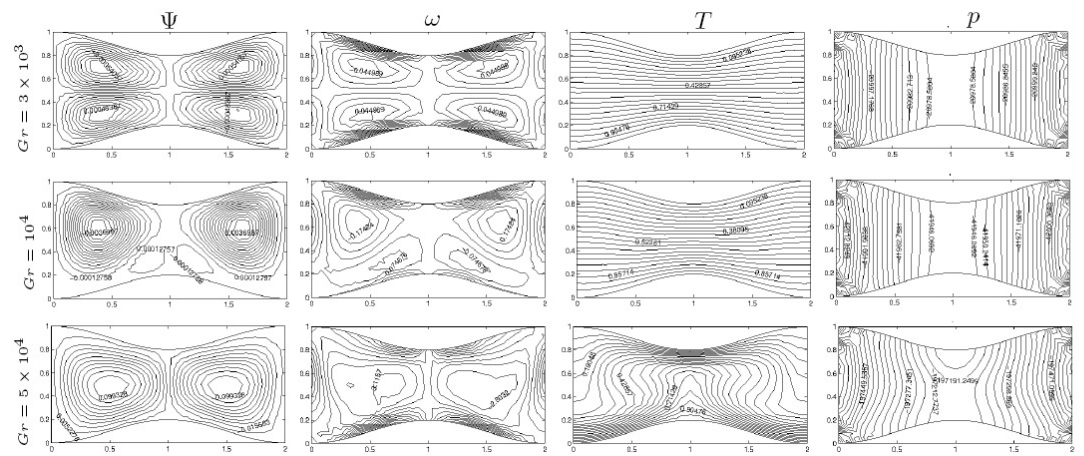

$\mathrm{M}=10$
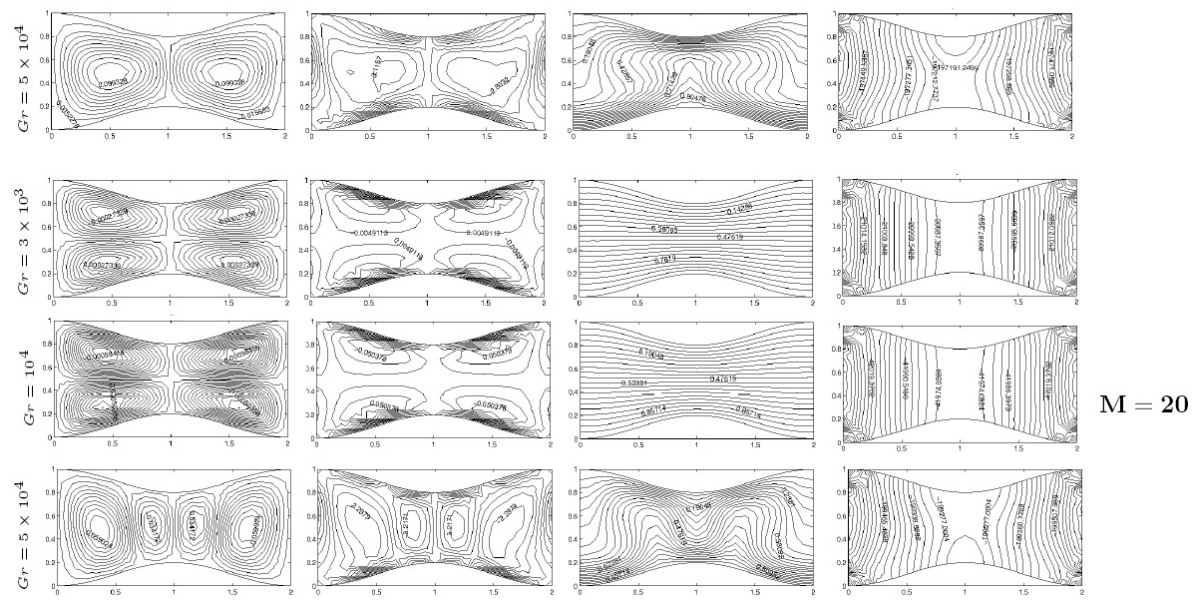

Figure 8: The effect of magnetic field on convection flow for $C R=40 \%$ and $A=2$.

\subsection{Magnetic field in the $x$-direction}

Next, the heat transfer on the MHD flow is considered in the constricted channel under the influence of horizontally applied magnetic field. The numerical results are depicted in terms of $\psi, \omega, T, p$ in Figure 9 for different Hartmann number values for fixed $C R=40 \%, G r=10^{4}$ and $A=4$. It is observed that the effect of horizontal magnetic field is nearly the same as the effect of magnetic field in the $y$-direction comparing with the results in Figure 7. Boundary layers on the top and bottom walls as $M$ increases are not pronounced much as in the case of vertically applied magnetic field since now these walls are parallel to the magnetic field. However, the fluid flows in terms of equally placed symmetric vortices with respect to axes in horizontally applied magnetic field. Isotherms and pressure are not affected with the direction of applied magnetic field. 

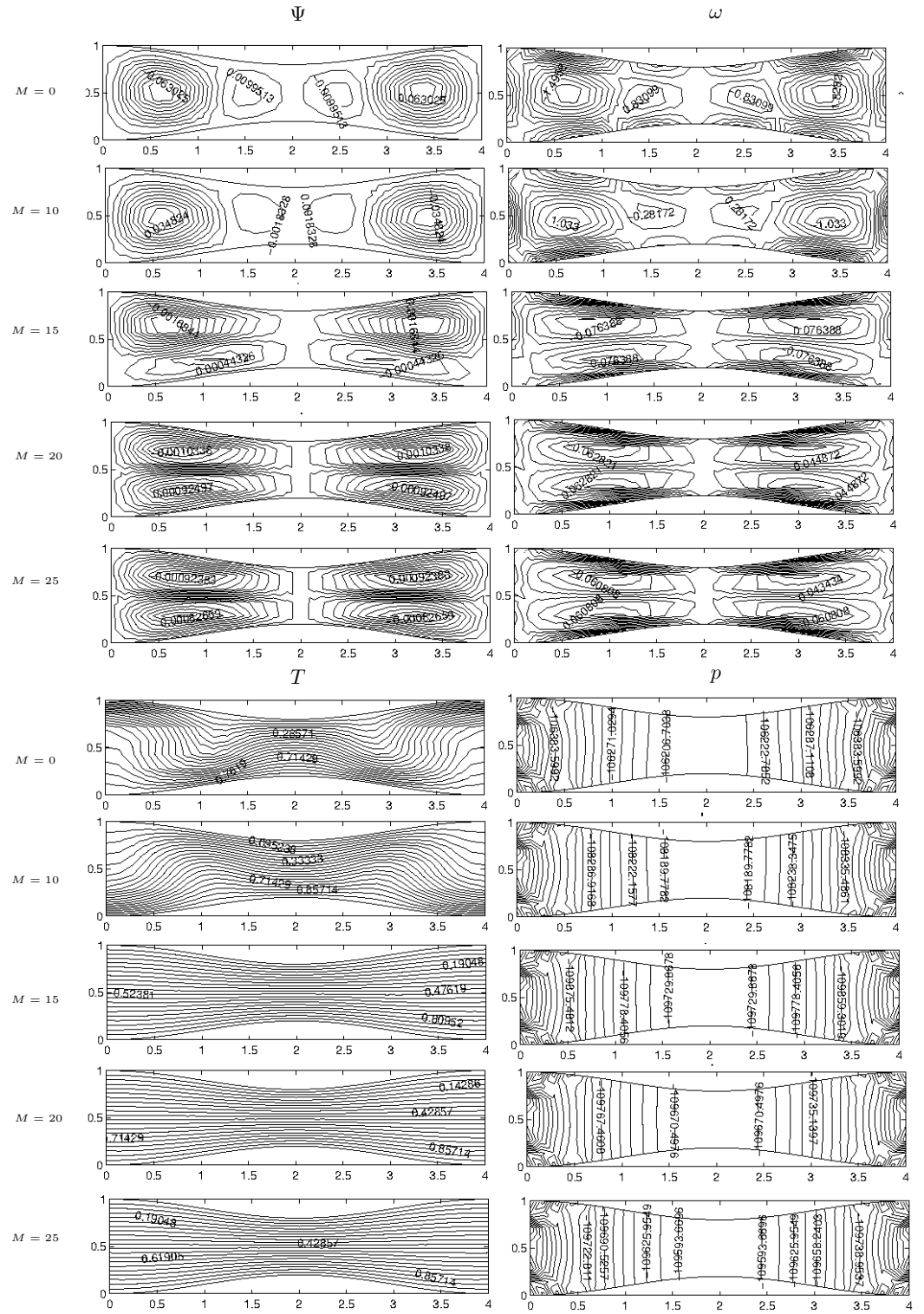

Figure 9: The effect of $M$ on the flow, temperature and pressure for $G r=10^{4}$, $C R=40 \%$ and $A=4$. 


\section{Conclusion}

RBF approximation with linear polynomials has been applied to MHD convection flow in constricted rectangular enclosure. Uniform magnetic field is applied either in the $x$ - or $y$-direction. The effects of Hartmann number, Grashof number, the constriction ratio on the flow, temperature, and pressure are investigated. The magnitude of the flow decreases as $M$ or $C R$ increases, however it increases as $G r$ increases. These are the well-known expected behaviors of MHD flow and convection flow, respectively. Increasing the horizontal length of the channel to $A$ produces $A$ number of convection rolls. An increase in the constriction ratio causes the retardation of the convection dominance. For a fixed constriction ratio, as $M$ increases the flow is divided symmetrically into more vortices with respect to axes.

\section{References}

[1] J.A. Kolodziej and J.K. Grabski, Application of the method of fundamental solutions and the radial basis functions for viscous laminar flow in wavy channel, Engineering Analysis with Boundary Elements, 57, 58-65 (2015).

[2] A. Al-Amiri, K. Khanafer, J. Bull and I. Pop, Effect of sinusoidal wavy bottom surface on mixed convection heat transfer in a lid-driven cavity, International Journal of Heat and Mass Transfer, 50, 1771-1780 (2007).

[3] S. Cetindag and M.K. Aktas, Numerical simulation of Rayleigh Benard convection in an enclosure: Effect of vibrating, In Proc. the World Congress on Engineering WCE 2014, July 2-4, London, UK.

[4] R. Nasrin, and S. Parvin, Hydromagnetic effect on mixed convection in a lid-driven cavity with sinusoidal corrugated bottom surface, International Communications in Heat and Mass Transfer, 38, 781-789 (2011).

[5] C.C. Wang and C.K. Chen, Mixed convection boundary layer flow on inclined wavy plates including the magnetic field effect, International Journal of Thermal Sciences, 44, 577-586 (2005).

[6] M.J. Colaço, G.S. Dulikravich, and H.R.B. Orlande, Magnetohydrodynamic simulations using radial basis functions, International Journal of Heat and Mass Transfer, 52, 5932-5939 (2009).

[7] D.C. Lo, High-resolution simulations of magnetohyrdodynamic free convection in an enclosure with a transverse magnetic field using a velocity- 
vorticity formulation, International Journal Communications in Heat and Mass Transfer, 37, 514-523 (2010).

[8] R. Mößner and U. Müller, A numerical investigation of three-dimensional magnetoconvection in rectangular cavities, International Journal of Heat and Mass Transfer, 42, 1111-1121 (1999).

[9] M. Gürbüz and M. Tezer-Sezgin, MHD Stokes flow in a smoothly constricted rectangular enclosure, Proceedings of Advances in Boundary Element \& Meshless Techniques XVII, BETEQ 2016, Ankara, Turkey, 73-78 (2016).

[10] U. Müller, and L. Bühler, Magnetofluiddynamics in channels and containers, Berlin, New York, 2001.

[11] C.S. Chen, C.M. Fan, P.H. Wen, The method of approximate particular solutions for solving certain partial differential equations, Numerical Methods for Partial Differential Equations, 28, 506-522 (2012).

[12] M. Gürbüz and M. Tezer-Sezgin, MHD Stokes flow in lid-driven cavity and backward-facing step channel, European Journal of Computational Mechanics, 24, 279-301 (2015).

[13] N.L. Gajbhiye and V. Eswaran, Numerical simulation of MHD flow and heat transfer in a rectangular and smoothly constricted enclosure, International Journal of Heat and Mass Transfer, 83, 441-449 (2015).

M. Tezer-Sezgin,

Department of Mathematics,

Middle East Technical University,

06800, Ankara, Turkey.

Email: munt@metu.edu.tr

Merve Gürbüz,

Department of Mathematics,

Middle East Technical University,

06800, Ankara, Turkey.

Email: mervegurbuz@baskent.edu.tr 